\title{
Autoimmune hemolytic anemia: transfusion challenges and solutions
}

\section{Melca M O Barros Dante M Langhi Jr José O Bordin}

Department of Clinical and Experimental Oncology, Universidade Federal de São Paulo, São Paulo, Brazil

This article was published in the following Dove Press journal:

International Journal of Clinical Transfusion Medicine

16 March 2017

Number of times this article has been viewed

\begin{abstract}
Autoimmune hemolytic anemia (AIHA) is defined as the increased destruction of red blood cells (RBCs) in the presence of anti-RBC autoantibodies and/or complement. Classification of AIHA is based on the optimal auto-RBC antibody reactivity temperatures and includes warm, cold-reactive, mixed AIHA, and drug-induced AIHA subtypes. AIHA is a rare disease, and recommendations for transfusion are based mainly on results from retrospective data and relatively small cohort studies, including heterogeneous patient samples or single case reports. In this article, we will review the challenges and solutions to safely transfuse AIHA patients. We will reflect on the indication for transfusion in AIHA and the difficulty in the accomplishment of immunohematological procedures for the selection of the safest and most compatible RBC units. Keywords: hemolytic anemia, RBC autoantibodies, autoimmunity, hemolysis, direct antiglobulin test
\end{abstract}

\section{Introduction}

Autoimmune hemolytic anemia (AIHA) is defined as the increased destruction of red blood cells (RBCs) in the presence of anti-RBC autoantibodies and/or complement. The annual incidence of AIHA range from 1 to 3 in 100,000 to 1 in 25,000 individuals. ${ }^{1-3}$ The variability likely reflects the use of different criteria for diagnosis of AIHA that often confounds comparison among studies. AIHA affects people of all ages, but it is more common in adults than in children. ${ }^{4}$ Symptoms and physical findings reflect the premature destruction of RBCs with inadequate compensation of bone marrow and the secondary effect of hemolysis. ${ }^{1,2}$ The diagnosis of AIHA is usually simple and based on the presence of hemolytic anemia and serological evidence of anti-RBC autoantibodies detected by the direct antiglobulin test (DAT). ${ }^{1}$

Since AIHA is a rare disease, treatment recommendations for patients with this condition, including transfusional support are based mainly on results from retrospective data and relatively small cohort studies, including heterogeneous patient samples or single case reports. Moreover, a frequent finding in immunohematology is the presence of anti-RBC autoantibodies without clinical symptoms of hemolysis.

Immune hemolytic anemias have been classified based on different criteria; however, AIHA is often classified by the optimal temperature at which the autoantibodies bind to patient's RBCs in vivo. In this context, the classification system includes warm AIHA (WAIHA), cold AIHA (CAIHA) (which includes cold agglutinin syndrome [CAS] and paroxysmal cold hemoglobinuria [PCH]), mixed AIHA (MAIHA), and drug-induced immune hemolytic anemia (DIIHA) (Table 1). In WAIHA, the autoantibodies present

Correspondence: José O Bordin Department of Clinical and Experimental Oncology, Universidade Federal de São Paulo, 824 Rua Diogo de Faria, CEP 04023-00I, São Paulo, SP, Brazil Email jobordin@unifesp.br 
Table I Classification of autoimmune hemolytic anemias

\begin{tabular}{|c|c|c|c|c|}
\hline \multirow[t]{2}{*}{ WAIHA } & \multicolumn{2}{|l|}{ CAIHA } & \multirow[t]{2}{*}{ MAIHA } & \multirow[t]{2}{*}{ DIIHA } \\
\hline & CAS & PCH & & \\
\hline Primary & Primary & Primary or chronic nonsyphilitic & Primary & True autoantibody \\
\hline Secondary & Secondary & Secondary & Secondary & Immune complex \\
\hline Lymphoproliferative disorders & Infections (M. pneumoniae, & Acute transient (viral infection) & & Drug adsorption \\
\hline Connective tissue diseases & $\mathrm{EBV}, \mathrm{CMV})$ & Chronic syphilitic & & Nonimmunological protein \\
\hline Solid tumors & Solid tumors & & & adsorption \\
\hline \multicolumn{5}{|l|}{ Myelodysplastic syndrome } \\
\hline Infections (HIV, HBV, HCV) & & & & \\
\hline
\end{tabular}

Abbreviations: WAIHA, warm autoimmune hemolytic anemia; CAlHA, cold autoimmune hemolytic anemia; MAlHA, mixed autoimmune hemolytic anemia; M. pneumoniae, Mycoplasma pneumoniae; DIIHA, drug-induced immune hemolytic anemia; CAS, cold agglutinin syndrome; $\mathrm{PCH}$, paroxysmal cold hemoglobinuria; $\mathrm{HBV}$, hepatitis B virus; $\mathrm{HCV}$, hepatitis $C$ virus; EBV, Epstein-Barr virus; CMV, cytomegalovirus.

in the serum react optimally with human $\mathrm{RBCs}$ at $37^{\circ} \mathrm{C}$, while CAIHA is mediated by cold autoantibodies exhibiting affinity for RBCs at temperatures below $37^{\circ} \mathrm{C}$, and MAIHA patients exhibit both cold- and warm-reactive autoantibodies. ${ }^{1-3}$

Cases of AIHA have also been classified by the presence or absence of various pathophysiologically associated underlying conditions. The term "primary or idiopathic AIHA" is applied when there is no recognizable underlying or associated condition, while the term "secondary AIHA" is detected as a manifestation of an underlying pathophysiological disorder. ${ }^{1}$

\section{Warm autoimmune hemolytic anemia}

WAIHA comprises $60 \%-90 \%$ of all AIHA cases. ${ }^{1-6}$ An Italian multicenter study including 308 patients with primary AIHA showed that $60 \%$ was classified as WAIHA. ${ }^{6}$

WAIHA is caused by warm autoantibodies that are predominantly IgG1 or IgG3 subclasses isolated or associated with complement fractions. This association with other clusters of immune origin reflects a generalized disturbance in the homeostasis of the immune system. ${ }^{1}$

The clinical features of WAIHA may be highly variable and depend on the rate of hemolysis, the degree of bone marrow compensation, and the presence of an underlying disorder. Usually, WAIHA patients insidiously develop anemic symptoms such as weakness, dizziness, fatigue, and dyspnea. ${ }^{1,2,5,7}$ Typically, autoimmune anemias are macrocytic because of the presence of marked reticulocytosis, but early in the presentation, transient reticulocytopenia may occur despite the presence of a normal or hyperplastic bone marrow. Reticulocytopenia may be seen due to the in vivo hemolysis of the reticulocytes or because of compromised bone marrow function. ${ }^{8}$ The blood film often reveals marked anisocytosis, polychromasia, and spherocytosis. Upon presentation, the hemolysis may be severe and, on occasion, may even be life-threatening. Typically, patients with
WAIHA exhibit a mild leukocytosis with neutrophilia but, occasionally, patients present with leucopenia, neutropenia, or thrombocytopenia due to associated immune-mediated neutrophil and/or platelet destruction. ${ }^{1}$ The laboratory parameters observed in patients with WAIHA include reduced serum haptoglobin level, increased indirect bilirubin level, and elevated lactate dehydrogenase (LDH) level. ${ }^{1,2}$ The presence of a positive DAT indicates that $\mathrm{RBC}$ autoantibodies and/or complement proteins, or both, are bound to the circulating RBCs in vivo.

\section{Cold autoimmune hemolytic anemia}

CAIHA caused by antibodies that optimally react at $4^{\circ} \mathrm{C}$ includes cases of CAS and PCH. ${ }^{9}$ AIHA caused by cold antibodies is relatively uncommon compared with the prevalence of WAIHA.

CAS comprises the majority of CAIHA cases, however it is relatively rare when compared to WAIHA, but it occurs much more frequently than $\mathrm{PCH}$. In an multicenter study with 308 patients with primary AIHA, 84 (27\%) patients had CAS, ${ }^{6}$ whereas in another series of 347 patients with acquired immune hemolytic anemias, including DIIHA, 54 (15.6\%) patients had CAS. ${ }^{2}$ The prevalence of CAS has been reported to vary from $7.7 \%$ to $15 \%$ in all cases of AIHA, with an incidence of 1 per million people per year. ${ }^{2,3,9,10}$

The primary CAS has been shown to be a clonal B-cell disorder, most often nonprogressive and clinically nonmalignant disease caused by a cold IgM $\kappa$ monoclonal autoantibody. The term "secondary chronic CAS" is reserved for those patients in that cold-antibody-mediated hemolytic anemia complicates an overt and well-defined malignant disease or infection. ${ }^{9,10,11}$ Idiopathic CAS generally occurs in middleaged or elderly adults, and predominantly affects women, while secondary CAS with infectious etiology, usually involves a young adult or adolescent with Mycoplasma pneumoniae or infectious mononucleosis (Epstein-Barr virus). 
CAS secondary to a malignant disease is far more uncommon than the primary disease. ${ }^{9,11}$

Patients with CAS present a mild to moderate chronic anemia. In addition to weakness, dizziness, fatigue, and dyspnea, clinical manifestations include cold-induced circulatory signals such as livedo reticularis, Raynaud disease, acrocyanosis, and cutaneous necrosis. Hemoglobinuria as evidence of intravascular hemolysis also may occur. ${ }^{2,9,11}$

The CAS laboratory parameters include increase in the indirect bilirubin level, elevated LDH level, and reduced haptoglobin level. The diagnosis of CAS is suggested by the autoagglutination of the patient's blood at room temperature that is exacerbated at $4{ }^{\circ} \mathrm{C}$, and is reverted at $37^{\circ} \mathrm{C}$. Patients with CAS present a positive DAT with anti-C3 or anti-C3d, but is negative with anti-IgG reagents. ${ }^{2,9,11}$

$\mathrm{PCH}$ is unusual in adults, but is one of the most common causes of acute AIHA in young children. $\mathrm{PCH}$ has been described following a viral illness or after vaccination. ${ }^{9,12}$

Clinically, the PCH is characterized by explosive intravascular hemolysis with hemoglobinuria and jaundice associated with abdominal pain, cutaneous mucosa pallor, fever, chills, and chronic headache, preceded by 1-2 weeks of a respiratory tract infection. Laboratory findings include increase in the indirect bilirubin level, elevated LDH level, reduced haptoglobin value, hemoglobinemia, and hemoglobinuria followed by an increment of serum levels of urea and creatinine if progression to renal failure occurs. $\mathrm{PCH}$ is a special type of hemolytic syndrome caused by a cold agglutinin called the Donath-Landsteiner antibody that corresponds to a polyclonal IgG biphasic antibody that bounds to RBCs at cold temperatures and produces intravascular hemolysis at normal body temperature. The complement is required for hemolysis, but plays little or no part in the fixation phase., ${ }^{9,12}$

\section{Mixed autoimmune hemolytic anemia}

MAIHA represents approximately $6 \%-8 \%$ of all AIHA reported in adults and it is extremely rare in children. ${ }^{13}$ Barcellini et al have recently reported that MAIHA accounted for $\sim 8 \%(24 / 308)$ of all primary AIHA investigated by the GIMEMA study, ${ }^{6}$ while Sokol et al found that MAIHA represented approximately $7 \%$ of cases of AIHA. ${ }^{13}$ In a study including a larger sample of patients, Mayer et al established diagnosis of MAIHA in only two $(<0.1 \%)$ out of 2,194 patients with warm autoantibody and concluded that the presence of cold antibodies with high thermal amplitude associated with warm antibodies may lead to confusion and misdiagnosis in some patients with AIHA. ${ }^{14}$
The serological findings in patients with MAIHA are the presence of cold autoantibodies with a high thermal amplitude $\left(>30^{\circ} \mathrm{C}\right)$ in association with warm autoantibodies. In contrast to the typical CAS, the IgM autoantibodies seen in MAIHA usually present low titles $(1: 64)$ at $4^{\circ} \mathrm{C}$ or less, but have great thermal amplitude, with reactivity to $37^{\circ} \mathrm{C}$. DAT shows positive reaction with anti-IgG and anti-C3d reagents, and the eluate contains the IgG autoantibody. ${ }^{9,13,14}$ MAIHA patients may also present clinically significant alloantibodies that are reactive at $37^{\circ} \mathrm{C}$, therefore the application of additional technical procedures may be necessary.

\section{Drug-induced immune hemolytic anemia}

DIIHA is a rare condition with a suggested incidence of approximately 1 in a million. DIIHA can be further subdivided depending on whether it is caused by drug-dependent antibodies or by drug-independent antibodies. Drug-dependent antibodies need the drug to detect in vitro reactions, while drug-independent antibodies react in vitro without the presence of any drug showing results that are indistinguishable from RBC autoantibodies. ${ }^{15,16}$ Petz and Garratty described four possible mechanisms of DIIHA: 1) immune complex; 2) drug adsorption; 3) nonimmunological protein adsorption; and 4) AIHA. ${ }^{2}$ The last mechanism occurs when the drug induced an autoantibody that reacts with normal RBCs (drug-independent), while the others occur by drugdependent antibodies resulting in positive DAT but negative eluate reaction. Garratty and Arndt described 142 drugs that they believe have reasonable evidence to support a drugassociated immune etiology for DIIHA or positive DATs. ${ }^{15}$

\section{Immunohematological assessment of patients with AIHA}

The immunohematological evaluation of AIHA confirms the clinical diagnosis, distinguishes among the various types, and identifies the safest blood for transfusion. The selection of blood for transfusion in AIHA is difficult because the presence of warm autoantibodies complicates routine pretransfusion testing and often demands the use of time-consuming, specialized techniques. The perfect communication between the blood bank and the clinical team is essential because the testing strategy is influenced by the patient's transfusion history as well as the urgency of the requirement for transfusion. The clinical team must have a general understanding of the principles and limitations of the immunohematological tests as well as the implications of their results to provide optimal transfusion therapy. 
The DAT is the standard procedure in demonstrating the presence of autoantibodies and/or complement proteins bound to circulating RBCs in vivo. A polyspecific antiglobulin reagent containing antibodies directed against human IgG and complement proteins (usually $\mathrm{C} 3$ ) is used to screen the patient's RBCs. When a RBC sample is positive with a polyspecific reagent, monospecific antisera with anti-IgG or anti-C3d are used to better define the pattern of the DAT. Monospecific antisera to IgM or IgA may also be used in selected cases. Since in most WAIHA cases, the autoantibody shows no RBC antigen specificity, the autoantibodies should be eluted from RBC after their detection by the DAT to potentially determine their specificities. ${ }^{1-3}$ Eluate negative results suggest the presence of DIIHA.

Generally, RBC autoantibodies are antigen nonspecific and react with all phenotyped panel cells but occasionally a broad specificity, usually related to the Rh system, is detected. Autoantibody specificities linked to other blood group antigen systems have been reported including Kell, Kidd, Duffy, and Diego blood group systems. ${ }^{1,5}$ It has been recommended that compatible blood must be transfused to AIHA patients carrying RBC-specific autoantibodies; however, some authors ignore the specificity of the autoantibody because some reports have not demonstrated significant differences in the survival of antigen-specific compatible RBCs used for transfusion, or have shown that the benefit was minimal. ${ }^{1,2,17,18}$

Patients with CAIHA present positive DAT result with anti-C3 or anti- $(\mathrm{C} 3+\mathrm{IgG})$ reagents but negative with anti$\mathrm{IgG}$ reagent. Table 2 summarizes the immunohematological findings in patients with various types of AIHA.

\section{Specialized immunohematological tests in patients with WAIHA}

Various approaches are useful to select the safest and most compatible RBC units for transfusion in patients with warm autoantibodies. Since time is a determining factor in blood transfusion, patients with no history of pregnancy or previous exposition to blood products are unlikely to carry a RBC alloantibody, therefore the pretransfusion tests can be simplified avoiding delay in the transfusion process. On the other hand, we should not be satisfied in offering "the least incompatible" transfusion to AIHA patients who show higher alloimmunization risk, therefore this term should be discouraged.

AIHA patients must be subjected to routine testing before blood transfusion including the $\mathrm{ABO}$ and $\mathrm{RhD}$ types as well as the antibody screening. The major technical problem encountered in patients with WAIHA is related to the presence of RBC alloantibodies which may be implicated in serious hemolytic transfusion reactions. A serological workup to investigate the presence of RBC alloantibodies includes testing the patient's serum with a selected panel of previously phenotyped reagent RBCs. When the autoantibody is less reactive than the alloantibody, the differences of the reaction against various cells of the panel permit the alloantibody identification. On the other hand, there is no assurance that an alloantibody will react more strongly than the autoantibody thus additional tests are necessary. Different approaches are available for detecting RBC alloantibodies including the patient's serum dilution before performing the compatibility test, and/or employing adsorption techniques which will remove the autoantibody but not the alloantibodies. Although these procedures for detection of RBC alloantibodies may be avoided for patients with no alloimmunization risk, the patient extended RBC phenotyping and genotyping are very beneficial and may be determined before the first RBC transfusion in order to facilitate future selection of blood units..$^{1,19}$

\section{Dilution technique}

The dilution technique should be used only in emergency situations because this technique is relatively easy and fast. Dilution is employed when a weakly reactive autoantibody and a strongly reactive alloantibody are present and the differences in the reactivity with several cells of the panel are

Table 2 Summary of immunohematological test findings in patients with AIHA

\begin{tabular}{llllllll}
\hline Type of AIHA & Ig & DAT poly & DAT mono anti-lgG & DAT mono anti-C3d & IAT & Eluate & Specificity \\
\hline WAIHA & $\operatorname{lgG}$ & + & + & + or - & + & IgG & Pan-agglutinin \\
& & & & & $+*$ & - & Rh/others (rare) \\
CAS & $\operatorname{lgM}$ & + & - & + & $++^{*}$ & - \\
PCH & $\operatorname{lgG}$ & + & - & + & $+*$ & - & P \\
DIIHA & $\operatorname{lgG}$ & + & + & + or - & - & + or - & Rh-like \\
\hline
\end{tabular}

Note: *Room temperature.

Abbreviations: AlHA, autoimmune hemolytic anemia; WAIHA, warm autoimmune hemolytic anemia; CAS, cold agglutinin syndrome; PCH, paroxysmal cold hemoglobinuria; DIIHA, drug-induced immune hemolytic anemia; Ig, immunoglobulin; DAT poly, direct antiglobulin test with polyspecific reagent (anti-gamma globulin and anticomplement); DAT mono anti-lgG, direct antiglobulin test with monospecific reagent anti-lgG; DAT mono anti-C3d, direct antiglobulin test with monospecific reagent anti-C3d; IAT, indirect antiglobulin test; + , positive; - , negative. 
evident. ${ }^{5,19-21}$ Leger and Garratty upon analyzing samples of 119 patients with autoantibodies, observed that the dilution technique was able to identify alloantibodies in only $19 \%$ of cases and concluded that although the 1-in-5 dilution approach is convenient, $27 \%$ of alloantibodies of potential clinical significance were not detected. ${ }^{21}$ Although this technique provides useful information, it is unreliable therefore more effective procedures must be performed.

The adsorption techniques remove the circulating autoantibody by serum incubation with autologous or allogeneic RBCs. After the adsorption procedure, the serum free of autoantibody is tested against previously typed panel RBCs for alloantibody identification. After the procedure, the adsorbed serum must be preserved for crossmatching test.

The warm autoadsorption technique is considered the best method for the detection of potentially significant alloantibodies because patient's RBCs are used to adsorb the autoantibodies from the patient's serum at $37^{\circ} \mathrm{C}$. Patient's RBCs are pretreated with a solution to remove some of the coating globulin and to increase their capacity to adsorb the autoantibodies from the serum. Autoadsorption is less laborious than allogeneic adsorption since only one sample of RBCs is necessary, and the treatment of RBCs with enzymes enhances the adsorption process. Another important advantage of the method is determining clinically significant alloantibodies against antigens of high frequency because autoantibodies are adsorbed without losing these alloantibodies which may occur in the allogeneic adsorption..$^{5,19-21}$ The limitations of the test consist of the time for the autoadsorption procedure to be effective, the limited volume of RBCs in patients with severe anemia, and that the procedure is not useful in patients transfused over the last 3 months because even a small amount of transfused RBCs may adsorb alloantibodies. ${ }^{5,19-21}$ A study using flow cytometry has shown that very small amounts of RBCs containing antigens are sufficient to adsorb the alloantibodies. $^{22}$

The second method of choice for alloantibodies identification in patients carrying autoantibodies is the allogeneic adsorption. If the patient extended phenotype or genotype are known compatible, RBCs may be used which facilitates the process. When RBC phenotype and/or genotype are unknown, the allogeneic adsorption should be performed by selecting three RBC samples with different phenotypes. Generally, the selected RBC samples are as follows: R1, R2, and rr; at least one sample lacking $\mathrm{K}$; randomly selected RBCs negative for $\mathrm{Jk}^{\mathrm{a}}, \mathrm{Jk}^{\mathrm{b}}, \mathrm{S}$, s; and RBCs treated with enzyme or ZZAP so that they are effectively negative for $\mathrm{Fy}^{\mathrm{a}}, \mathrm{Fy}^{\mathrm{b}}, \mathrm{M}$, and N. ${ }^{19-21}$
This allogeneic adsorption technique has some limitations because it is labor-intensive and the expected time required for adsorption varies significantly depending on the method, the number of RBC samples, and the number of the adsorptions executed to totally remove the autoantibody. The great pitfalls with allogeneic adsorptions is that, if there is an antibody to a high-frequency antigen, it will be adsorbed by allogeneic RBCs and will not be detectable, making weak alloantibodies possibly not detected. ${ }^{19-21}$

The final step in the selection of blood for transfusion is the crossmatching test that consists of the incubation of patient's serum with donor's RBCs. When alloantibodies are found, RBC units negative for the corresponding antigen should be selected. The most appropriate method for selecting units for WAIHA patients is to use the adsorbed serum and cross it with previously typed units, in that way completely compatible and safe blood will be transfused. In contrast, in situations without necessary time for adsorption procedures, the autoantibody present in serum typically attaches to all RBCs, making the blood unit incompatible, therefore some transfusion services choose the so-called "least incompatible" unit even if no specificity of the reactions can be ascertained. There is no clinical or laboratory scientific evidence of increased in vivo RBC survival of less reactive units (least incompatible) when compared to laboratory strongly reactive units. Considering the lack of sound scientific data, many past recommendations regarding $\mathrm{RBC}$ transfusions in patients with AIHA are questionable therefore we recommend the use of the safest blood unit selected by pretransfusion technical procedures. ${ }^{19}$ Figure 1 describes a suggested algorithm for providing RBC transfusion to AIHA patients.

Extended phenotyping is defined as typing RBCs for numerous clinically relevant antigens (ie, D, C, E, c, e, K, Jka, Jkb, Fya, Fyb, S, and s). ${ }^{19,23}$ Determining complete phenotypes of patients with WAIHA helps to identify possible $\mathrm{RBC}$ alloantibodies and to select appropriate RBCs for the adsorption technique. When extended RBC phenotyping is performed, the blood bank is able to provide prophylactic antigen-matched blood preventing RBC alloimmunization, and avoiding future acute or delayed hemolytic transfusion reactions. In a study including 20 patients with WAIHA (eight patients had preexisting alloantibodies), a phenotype was determined in 12 of them, and a total of 149 prophylactic antigen-matched RBC units were transfused. None of the patients developed new alloantibodies, the expected posttransfusion increments in hemoglobin were achieved, and no signs of hemolytic transfusion reactions were noted. ${ }^{23}$ However, in eight patients, phenotypes were completely 


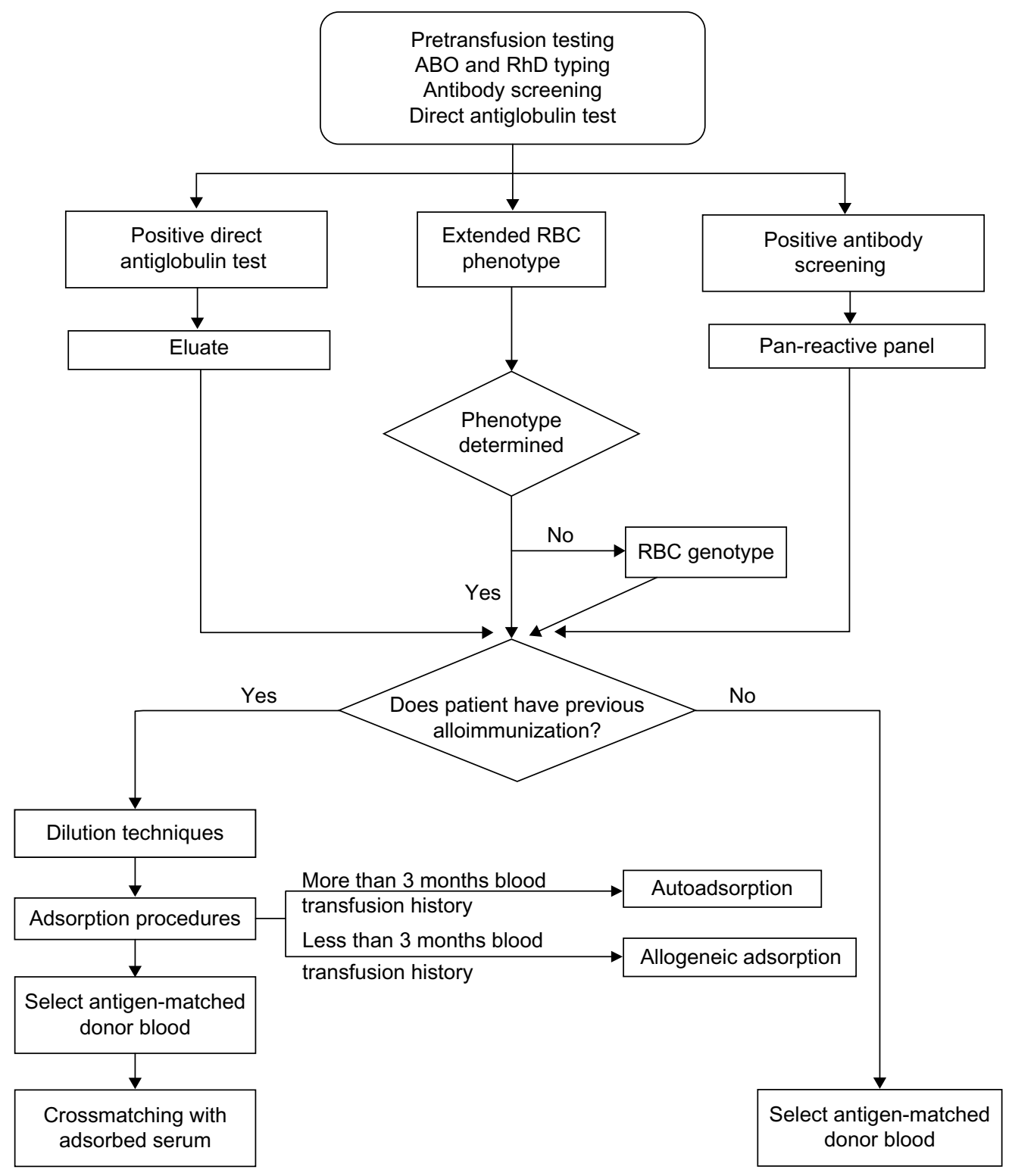

Figure I Algorithm for RBC transfusion in patients with WAIHA.

Abbreviations: RBC, red blood cell; WAIHA, warm autoimmune hemolytic anemia.

or partially indeterminate showing that determining the extended RBC phenotype is technically difficult or even impossible when the surface of the patient's RBCs is coated with autoantibodies. Defining some antigen phenotypes may be accomplished by use of the low-protein (monoclonal IgM), or pretreating patient's RBCs with a solution, such as chloroquine, to enable IgG dissociation. In some series, $40 \%-50 \%$ of the patients still remain with autoantibodies in their RBCs, and the extended RBC phenotype in WAIHA patients is determined in $50 \%-60 \%$ of the cases..$^{2-5,19,23}$ None of the patients developed new alloantibodies, the expected posttransfusion increments in hemoglobin were achieved, and no signs of hemolytic transfusion reactions were noted. ${ }^{23}$ Whether implementation of this approach is cost-effective and feasible at many hospitals and blood centers has not been determined yet. The extended RBC phenotype is better performed if the patient has not received blood transfusion over the last 3 months.

Over the last decades, the genes encoding 29 of the 30 blood group systems have been cloned and sequenced; therefore, the molecular basis associated with most RBC antigens has been determined. By genotyping, the apparent RBC phenotype is deduced through the analysis of the individual genomic DNA. The commercial development of the polymerase chain reaction (PCR)-based technology makes the development of diverse techniques of molecular biology that are used in the transfusion medicine. The first blood group genotyping assays consisting of PCR-restriction fragment 
length polymorphism and PCR-allele-specific assays require post-PCR sample manipulation and gel electrophoresis to separate and analyze the pattern of fragments. Other assays consisting of the real-time PCR with automated fluorescence discrimination of alleles, and amplification of multiple blood group systems in one tube, such as the multiplex PCR arrays, are also available. ${ }^{24}$

The molecular methods do not suffer interference by the $\mathrm{RBC}$ autoantibody constituting a great advantage when compared with methods of RBC phenotyping for patients with AIHA. After transfusion, the presence of donor RBCs in peripheral blood often makes RBCs phenotyping by agglutination techniques inaccurate. Genotyping overcomes this limitation and offers an alternative approach for patients with WAIHA. ${ }^{24}$ The limitations for universal application of molecular assays include the requirement of highly trained personnel and the expensive cost of the tests.

\section{Specialized immunohematological tests in patients with CAIHA}

Compatibility tests in patients with cold antibodies are less laborious because cold autoantibodies are not reactive at $37^{\circ} \mathrm{C}$ allowing an easier identification of clinically significant alloantibodies that react at this temperature. Pretransfusion testing used in CAS and $\mathrm{PCH}$ are similar since all technical procedures can be performed strictly at $37^{\circ} \mathrm{C}$. Cold autoantibodies may cause autoagglutination at room temperature resulting in erroneous determinations of the $\mathrm{ABO}$ and $\mathrm{RhD}$ types. RBCs should be incubated at $37^{\circ} \mathrm{C}$ for 10 minutes and washed several times in saline at the same temperature, patient's serum and reagent RBCs must also be warmed to $37^{\circ} \mathrm{C}$ before mixing, and the final test has to be centrifuged at $37^{\circ} \mathrm{C} \cdot{ }^{9,11,19,25}$

The compatibility testing may be performed at $37^{\circ} \mathrm{C}$ even in situations in which the patient has been recently transfused, eliminating the need for adsorption tests that may delay the $\mathrm{RBC}$ transfusion. Rarely, clinically significant $\mathrm{RBC}$ alloantibodies that react at temperatures below $37^{\circ} \mathrm{C}$ may not be detected. ${ }^{11,19}$

Cold-antibody adsorption is an alternative method in $\mathrm{CAS}$ and $\mathrm{PCH}$. If the transfusion service cannot ensure the performance of testing at $37^{\circ} \mathrm{C}$, one or two autoadsorption procedures often satisfactorily remove the cold agglutinins. Recently, transfused patients can undergo allogeneic test adsorption by the same manner as warm autoantibodies in patients with WAIHA. ${ }^{19}$

Another way to perform compatibility testing in patients with CAS is the inactivation of cold IgM agglutinins with the use of 2-mercaptoethanol or dithiothreitol. The use of sulfhydryl reagents denature IgM molecules. ${ }^{10}$

The final step in the selection of blood for transfusion is the crossmatching testing that consists of the incubation of patient's serum with donor's RBCs. In patients carrying cold autoantibodies, this procedure must be performed at $37^{\circ} \mathrm{C}$. If there is detection of clinically significant alloantibodies, RBCs that do not contain the corresponding antigen should be selected.

Autoantibodies in CAS are most often directed against the Ii blood group system. Approximately 90\% of cold agglutinins are anti-I specific, while most of the remaining $10 \%$ show specificity for the I antigen. Anti-I specificity is observed in both the primary disease and the secondary disease to $M$. pneumoniae or lymphoproliferative diseases. Anti-I specificity is typically associated with mononucleosis, but it can also be found in lymphomas and cytomegalovirus infection. Providing transfusion with I antigen negative $\mathrm{RBCs}$ is not easy because of its rarity and also because this strategy may delay the transfusion and has not proved to be clinically beneficial. The Donath-Landsteiner antibody found in PCH usually shows specificity for the P antigen. Effective transfusion to patients with $\mathrm{PCH}$ has been reported by various authors and, almost certainly, the transfusions contained $\mathrm{P}(+)$ blood. ${ }^{9,19,25}$

\section{Transfusion in autoimmune hemolytic anemia}

$\mathrm{RBC}$ transfusion is often necessary in the management of patients with AIHA. Patients with severe anemia should receive $\mathrm{RBC}$ transfusion to maintain their hemoglobin at a clinically acceptable level at least until other therapeutic strategies come into play which curtail the hemolysis, and the patient's bone marrow compensates the rapid $\mathrm{RBC}$ destruction. The recommendation for blood transfusion is mainly based on the severity of the hemolysis, the progression of the anemia and, more importantly, to the associated clinical findings. RBC transfusion may be necessary particularly for the symptomatic elderly, for patients with coronary artery disease with increased risk of cardiac or cerebral events, and life-threatening situations related to the anemia. ${ }^{13,14,17-20}$ First-line treatment of individuals with AIHA consists of glucocorticoids that often results in early improvement of the hemolytic state interrupting the hemoglobin level decrease usually 24-72 hours after therapy has been started. Another possible initial treatment for severe or refractory patients is the use of anti-CD20 monoclonal antibody (rituximab) that may rapidly reduce the hemolysis making RBC transfusion unnecessary. ${ }^{1}$ 
Blood transfusion for patients with AIHA presents a unique set of potential problems because of the relatively short survival time of the transfused RBCs, the masking effect of the presence of RBC alloantibodies by autoantibodies, and the need for complex pretransfusion immunohematological compatibility tests. It has been demonstrated that when incompatibility is due solely to the presence of the RBC autoantibodies, the survival time of transfused allogeneic RBCs is approximately the same as that of autologous RBCs, therefore $\mathrm{RBC}$ transfusion provides only some temporary benefit. ${ }^{1,19,26}$ Yürek et al observed no significant hemolytic transfusion reactions due to auto- and/or alloantibodies in 32 AIHA patients during or after RBC transfusion. ${ }^{27}$ In this latter study, three patients remained untransfused or had a postponed transfusion and died.

There are no reports that definitively demonstrate significant exacerbation of hemolysis in transfused patients with AIHA, therefore it has been considered that precautions surrounding blood transfusion in AIHA are overestimated. Park et al reported similar increments in the hemoglobin level in patients with RBC autoantibodies only (1.40-1.70 g/dL), with alloantibodies only $(1.20-1.60 \mathrm{~g} / \mathrm{dL})$, or no antibodies $(1.40-1.55 \mathrm{~g} / \mathrm{dL})$ for 7 days following transfusion of $10 \mathrm{~mL}$ RBCs per kg, without any associated increase in hemolysis risk. ${ }^{17}$ Das et al found a median hemoglobin value increment of $0.88 \mathrm{~g} / \mathrm{dL}$ after transfusion of each unit of RBC concentrate in patients with severe AIHA. ${ }^{28}$

Patients with WAIHA usually have broadly reactive autoantibodies that generally are pan-agglutinins which react with antigens present on all potential donor RBCs. Such autoantibodies often mask the concomitant presence of RBC alloantibodies developed as a result of previous transfusions or pregnancies causing the most important technical problem faced by the blood bank service when selecting blood for transfusion in patients with WAIHA. This concern relates to the fact that RBC alloantibodies may cause serious acute or delayed hemolytic transfusion reactions in recipients. ${ }^{1,26}$

The prevalence of RBC alloantibodies in patients with WAIHA ranges from $7.5 \%$ to $54 \%$ (Table 3 ). ${ }^{17,23,27-33}$ The majority of studies reporting on the high frequency of coexisting alloantibodies and autoantibodies mostly describe patients with serological positive results rather than with clinically confirmed WAIHA. We have recently reported a RBC alloantibody prevalence of $11.1 \%$ in Brazilian patients with true AIHA. ${ }^{30}$ The clinical physician attending patients with AIHA should understand that the aforementioned aspects of pretransfusion compatibility testing on AIHA patients are complex, and thus consider that a longer period of
Table 3 Prevalence of RBC alloantibodies in patients with AIHA

\begin{tabular}{|c|c|c|c|}
\hline Author & $\mathbf{N}$ & Prevalence (\%) & Alloantibody \\
\hline Yurek et $\mathrm{al}^{27}$ & 36 & $19.4^{*}$ & Anti-c, anti-E, anti-Jka \\
\hline Park et $\mathrm{al}^{17}$ & 161 & 54 & - \\
\hline Das et $\mathrm{al}^{28}$ & 14 & 7.5 & Anti-C \\
\hline Yu et $\mathrm{a}^{29}$ & 61 & 29.5 & - \\
\hline Barros et $\mathrm{a}^{30}$ & 36 & II.I & Anti-E, Anti-C \\
\hline Das et $\mathrm{al}^{31}$ & 23 & 30.4 & Anti-C, Anti-E \\
\hline Shirey et $\mathrm{a}^{23}$ & 20 & 40 & $\begin{array}{l}\text { Anti-D, anti-E, anti-C, } \\
\text { anti-Cw, anti-K, anti- } \\
J^{2} k^{\mathrm{a}} \text {, anti-Jk }{ }^{\mathrm{b}}\end{array}$ \\
\hline Branch et $\mathrm{al}^{20}$ & $647 * *$ & 32 & - \\
\hline Leger et $\mathrm{a}^{21}$ & 263 & 40 & $\begin{array}{l}\text { Anti-D, anti-E, anti-C, } \\
\text { anti-c, anti-K, anti-Jk } \\
\text { anti-Jk }^{\mathrm{b}} \text {, anti-Fy }\end{array}$ \\
\hline Issit et $\mathrm{al}^{32}$ & 138 & 43 & - \\
\hline Laine et $\mathrm{al}^{33}$ & 109 & 38 & - \\
\hline
\end{tabular}

Notes: $* 8.3 \%$ were clinically significant alloantibodies; $* *$ mean of collective data from 1982 to 1999 . "-" indicates no data.

Abbreviations: RBC, red blood cell; AlHA, autoimmune hemolytic anemia.

time is necessary for the blood bank to perform the complete panel of immunohematological tests which are necessary to guarantee the best RBC product for transfusion.

\section{Indications for transfusion in AIHA}

The RBC transfusion protocols designed to indicate transfusion in patients with AIHA are not significantly different from those employed for anemic patients without AIHA, and are mainly related to the onset and the severity of the anemia. In addition to a careful clinical evaluation, the results of the laboratory tests offer some guidance for the RBC transfusion necessity. Serial determinations of hemoglobin and hematocrit levels as well as other secondary laboratory tests such as indirect bilirubin, $\mathrm{LDH}$, and haptoglobin must be performed at regular intervals according to the severity of the hemolysis.

AIHA patients whose anemia has developed slowly are relatively asymptomatic and generally do not require RBC transfusions. On the other hand, transfusion should be indicated when AIHA patients present signs of hypoxemia such as angina, cardiac, or neurological symptoms reflected by lethargy, weakness, sleepiness, and/or mental confusion. These symptoms generally occur in patients with very profound anemia showing hemoglobin levels below $5 \mathrm{~g} / \mathrm{dL}$ (Table 4). ${ }^{1,6,17,18,28,34}$ In mild or moderate anemias $(\mathrm{Hb}>8 \mathrm{~g} /$ dL) RBC transfusion is rarely necessary or desirable, as symptoms generally are controlled with appropriated therapy. For patients showing hemoglobin level between 5 and $8 \mathrm{~g} / \mathrm{dL}$ the clinical decision for transfusion is more difficult. These patients should be under close clinical and laboratorial observation until the critical symptoms of anemia are controlled and the hemolysis is not progressing. The progression to 
Table 4 Level of hemoglobin and RBC transfusion in patients with AlHA

\begin{tabular}{|c|c|c|c|}
\hline Author & $\mathbf{N}$ & $\mathrm{Hb}(\mathrm{g} / \mathrm{dL})$ & $\begin{array}{l}\text { Mean of RBC units } \\
\text { transfused per } \\
\text { patient }\end{array}$ \\
\hline Park et al ${ }^{17}$ & 161 & $7.5(2.1-12.5)$ & $2(I-5)$ \\
\hline Barcellini et $\mathrm{a}^{6}$ & 115 & $<8$ & - \\
\hline Das et $\mathrm{a}^{28}$ & 15 & $3.8-6.5$ & 1.86 \\
\hline Roumier et $\mathrm{al}^{34}$ & 39 & $<8$ & 3.9 \\
\hline Wikman et $\mathrm{al}^{18}$ & 20 & $3.9-9.9$ & $2(I-5)$ \\
\hline
\end{tabular}

Note: "-" indicates no data.

Abbreviations: RBC, red blood cell; AlHA, autoimmune hemolytic anemia; $\mathrm{Hb}$, hemoglobin.

anemic hypoxia signals indicates that $\mathrm{RBC}$ transfusions are necessary until the therapeutic measures become effective. In such patients, RBC transfusion should not be delayed because fatalities have been reported when withholding or not administering transfusion. ${ }^{8,27}$ Barcellini et al transfused 115 patients with severe AIHA $(\mathrm{Hb}<8 \mathrm{~g} / \mathrm{dL})$ and observed that approximately one-third (38/115) of the patients were unresponsive, especially those with lower hemoglobin level $(\mathrm{Hb}<6 \mathrm{~g} / \mathrm{dL}[43 \%]$ vs $\mathrm{Hb}=6.1-8 \mathrm{~g} / \mathrm{dL}$ [25\%], $P=0.004)$. The authors also reported that the unresponsiveness to transfusions was not reasonably related to the presence of RBC alloantibodies. Roumier et al transfused 39/60 (65\%) patients with WAIHA because of anemia $(\mathrm{Hb}<8 \mathrm{~g} / \mathrm{dL})$ with symptoms of poor tolerance such as dizziness and/or comorbidities. Although the mean number of packed RBC units was 3.9 at the AIHA onset and 10 during clinical follow-up, no transfusion-related adverse events were observed. ${ }^{34}$ Das et al transfused 14 WAIHA patients with severe anemia reflected by hemoglobin values ranging from 3.3 to $6.5 \mathrm{~g} / \mathrm{dL}$, and reported that only one individual presented chills with no other adverse effects of transfusion observed. ${ }^{28}$

The association of WAIHA with other illnesses has also to be considered before indicating transfusion because patients with cardiac disease show less tolerance to acute anemia thus $\mathrm{RBC}$ transfusions must be performed to maintain the hemoglobin at a clinically acceptable level until other measures to stop the hemolysis can take effect.

The kinetics of RBC destruction in patients with WAIHA constantly describe an exponential curve of decay, indicating that the number of cells removed over a unit of time is a percentage of the number of cells present at the start of this time interval; consequently, if a larger volume of RBCs were transfused in one unit of time, hemolysis may increase..$^{35}$ Hemoglobinemia and hemoglobinuria following transfusion in a patient with AIHA have generally been attributed to an increased rate of hemolysis, although they may more commonly occur as a result of the increase in the total mass of
RBCs available for destruction. It has been suggested that in these situations, the blood volume transfused should be the smallest amount required to maintain adequate oxygen delivery, and not necessarily to reach an arbitrary defined hemoglobin level. The total volume transfused should not exceed $1 \mathrm{~mL} / \mathrm{kg}$ /hour. Some studies have demonstrated that $\mathrm{RBC}$ transfusion did not result in a significant increase in hemolysis and there are no reports that definitively demonstrate significant exacerbation of hemolysis in patients with true AIHA. ${ }^{17,26-28}$

Very rapid transfusion of cold blood components in resuscitation may cause hazardous hypothermia, however blood warming is seldom necessary and associated with considerable risks to the patient. The indication to warm blood units before transfusing patients with CAIHA is controversial, and there are few data indicating its effectiveness. In addition, no well-designed studies were conducted to evaluate the survival of transfused RBCs at different temperatures. When appropriately indicated, the heating of blood should be monitored and controlled and the patient's extremity chosen for infusion should be kept warm. ${ }^{10,17,36}$

\section{Disclosure}

The authors report no conflicts of interest in this work.

\section{References}

1. Barros MM, Blajchman MA, Bordin JO. Warm autoimmune hemolytic anemia: recent progress in understanding the immunobiology and the treatment. Transfus Med Rev. 2010;24(3):195-210.

2. Petz LD, Garratty G. Acquired Immune Hemolytic Anemia. New York: Churchill Livingstone; 1980.

3. Sokol RJ, Hewitt S, Stamps BK. Autoimmune haemolysis: an 18-year study of 865 cases referred to a regional transfusion centre. $\mathrm{Br} \mathrm{Med} J$ (Clin Red Ed). 1981;282(6281):2023-2027.

4. Habibi B, Homberg JC, Schiason G, Salmon C. Autoimmune hemolytic anemia in children. A review of 80 cases. Am J Med. 1974;56(1): 61-69.

5. Packman $\mathrm{CH}$. Hemolytic anemia due to warm autoantibodies. Blood Rev. 2008;22(1):17-31.

6. Barcellini W, Fattizzo B, Zaninoni A, et al. Clinical heterogeneity and predictors of outcome in primary autoimmune hemolytic anemia: a GIMEMA study of 308 patients. Blood. 2014;124(19):2930-2936.

7. Genty I, Michel M, Hermine O, Schaeffer A, Godeau B, Rochant H Caractéristiques des anémies hémolytiques auto-immunes de l'adulte: Analyse rétrospective d'une série de 83 patients [Characteristics of autoimmune hemolytic anemia in adults: retrospective analysis of 83 cases]. Rev Méd Interne. 2002;23(11):901-909. French.

8. Conley CL, Lippman SM, Ness PM, Petz LD, Branch DR, Gallagher MT. Autoimmune hemolytic anemia with reticulocytopenia and erythroid marrow. N Engl J Med. 1982;306(5):281-286.

9. Petz LD. Cold antibody autoimmune hemolytic anemias. Blood Rev. 2008;22(1):1-15.

10. Dacie J. The auto-immune haemolytic anaemias: introduction. In: Dacie J, editor. The Haemolytic Anaemias, Vol. 3. London: Churchill Livingstone; 1992:1-5.

11. Berentsen S, Tjonnfjord GE. Diagnosis and treatment of cold agglutinin mediated autoimmune hemolytic anemia. Blood Rev. 2012;26(3) $107-115$. 
12. Heddle NM. Acute paroxysmal cold hemoglobinuria. Transfus Med Rev. 1989;3(3):219-229.

13. Sokol RJ, Hewitt S, Stamps BK. Autoimmune hemolysis: mixed warm and cold antibody type. Acta Haematologica. 1983;69(4):266-274.

14. Mayer B, Yurek S, Kiesewetter H, Salama A. Mixed-type autoimmune haemolytic anemia: differential diagnosis and a critical review of reported cases. Transfusion. 2008;48(10):2229-2234.

15. Garratty G, Arndt PA. Drugs that have been shown to cause drug-induced immune hemolytic anemia or positive direct antiglobulin tests: some interesting findings since 2007. Immunohematology. 2014;30(2):66-79.

16. Garratty G. Drug-induced immune hemolytic anemia. Hematology Am Soc Hematol Educ Program. 2009;73-79.

17. Park SH, Choe WH, Kwon SW. Red blood cell transfusion in patients with autoantibodies: is it effective and safe without increasing hemolysis risk? Ann Lab Med. 2015;35(4):436-444.

18. Wikman A, Axdorph U, Gryfelt G, Gustafsson L, Björkholm M, Lundahl J. Characterization of red cell autoantibodies in consecutive DAT-positive patients with relation to in vivo haemolysis. Ann Hematol. 2005;84(3):150-158.

19. Petz LD. A physician's guide to transfusion in autoimmune haemolytic anaemia. Br J Haematol. 2004;124(6):712-716.

20. Branch DR; Petz, LD. Detecting alloantibodies in patients with autoantibodies. Transfusion. 1999;39(1):6-10.

21. Leger RM, Garratty G. Evaluation of methods for detecting alloantibodies underlying warm autoantibodies. Transfusion. 1999;39(1):11-16.

22. Laine EP, Leger RM, Arndt PA, Calhoun L, Garratty G, Petz LD. In vitro studies of the impact of transfusion on the detection of alloantibodies after autoadsorption. Transfusion. 2000;40(11):1384-1387.

23. Shirey, RS, Boyd JS, Parwani AV, Tanz WS, Ness PM, King KE. Prophylactic antigen-matched donor blood for patients with warm autoantibodies: an algorithm for transfusion management. Transfusion. 2002;42(11):1435-1441.

24. Reid ME, Denomme GA. DNA-based methods in the immunohematology reference laboratory. Transfus Apher Sci. 2011;44(1):65-72.
25. Swiecicki PL, Hegerova LT, Gertz MA. Cold agglutinin disease. Blood. 2013;122(7):1114-1121.

26. Salama A, Berghöfer H, Mueller-Eckhardt C. Red blood cell transfusion in warm-type autoimmune haemolytic anaemia. Lancet. 1992; 340(8834-8835):1515-1517.

27. Yürek S, Mayer B, Almahallawi M, Pruss A, Salama A. Precautions surrounding blood transfusion in autoimmune haemolytic anaemias are overestimated. Blood Transfus. 2015;13(4):616-621.

28. Das SS, Zaman RU, Safi M. Incompatible blood transfusion: challenging yet lifesaving in the management of acute severe autoimmune hemolytic anemia. Asian J Transfus Sci. 2014;8(2):105-108.

29. Yu Y, Sun XL, Ma CY, et al. Serological characteristics and transfusion efficacy evaluation in 61 cases of autoimmune hemolytic anemia. Zhongguo Shi Yan Xue Ye Xue Za Zhi. 2013;21(5):1275-1279.

30. Barros MM, Yamamoto M, Figueiredo MS, et al. Expression levels of CD47, CD35, CD55, and CD59 on red blood cells and signal-regulatory protein-alpha,beta on monocytes from patients with warm autoimmune hemolytic anemia. Transfusion. 2009;49(1):154-160.

31. Das SS, Chaudhary R. Utility of adsorption techniques in serological evaluation of warm autoimmune haemolytic anaemia. Blood Transfus. 2009;7(4):300-304.

32. Issitt PD, Combs MR, Bumgarner DJ, Allen J, Kirkland A, MelroyCarawan $\mathrm{H}$. Studies of antibodies in the sera of patients who have made red cell autoantibodies. Transfusion. 1996;36(6):481-486.

33. Laine ML, Beattie KM. Frequency of alloantibodies accompanying autoantibodies. Transfusion. 1985;25(6):545-546.

34. Roumier M, Loustau V, Guillaud C, et al. Characteristics and outcome of warm autoimmune hemolytic anemia in adults: new insights based on a single-center experience with 60 patients. Am J Hematol. 2014;89(9): E150-E155.

35. Rosenfield RE, Jagathambal. Transfusion therapy for autoimmunehemolytic anemia. Semin Hematol. 1976;13(4):311-321.

36. Iserson KV, Huestis DW. Blood warming: current applications and techniques. Transfusion. 1991;31(6):558-571.
International Journal of Clinical Transfusion Medicine

\section{Publish your work in this journal}

International Journal of Clinical Transfusion Medicine is an international, peer-reviewed, open access, online journal publishing clinicalexperimental, policy-making and evidence-based practices of all topics pertaining to clinical transfusion medicine. Original research, short reports, reviews, case reports and commentaries are invited.

\section{Dovepress}

The manuscript management system is completely online and includes a very quick and fair peer-review system, which is all easy to use. Visit http://www.dovepress.com/testimonials.php to read real quotes from published authors. 